\title{
Whole-Genome Sequences of the Severe Acute Respiratory Syndrome Coronavirus-2 obtained from Romanian patients between March and June of 2020
}

\section{Authors:}

1. Mihaela Lazar, Cantacuzino Military-Medical Research and Development National Institute Bucharest, Romania

2. Odette Popovici, Romanian National Institute Of Public Health, Bucharest, Romania

3. Barbara Mühlemann, Institute of Virology, Charité - Universitätsmedizin Berlin

4. Tim Durfee - DNASTAR Inc, USA

5. Razvan Stan - Cantacuzino Military-Medical Research and Development National Institute Bucharest, Romania

Corresponding author's name and mailing address, telephone number, and e-mail address: Mihaela Lazar, Cantacuzino Military-Medical Research and Development National Institute, Splaiul Independentei 103, Bucharest, Romania, lazar.mihaela@ cantacuzino.ro, +40 213069116.

\section{Abstract}

Impact of mutations on the evolution of Severe Acute Respiratory Syndrome Coronavirus-2 (SARS-CoV-2) are needed for ongoing global efforts to track and trace the current pandemic, in order to enact effective prevention and treatment options. SARS-Co-V-2 viral genomes were detected and sequenced from 18 Romanian patients suffering from coronavirus disease-2019. Viral Spike S glycoprotein sequences were used to generate model structures and assess the role of mutations on protein stability. We integrated the phylogenetic tree within the available European SARS-Co-V-2 genomic sequences. We further provide an epidemiological overview of the pre-existing conditions that are lethal in relevant Romanian patients. Non-synonymous mutations in the viral Spike glycoprotein relating to infectivity are constructed in models of protein structures. Continuing search to limit and treat SARS-CoV-2 benefit from our contribution in delineating the viral Spike glycoprotein mutations, as well as from assessment of their role on protein stability or complex formation with human receptor angiotensin-converting enzyme 2. Our results help implement and extend worldwide genomic surveillance of coronavirus disease-2019.

Keywords: SARS-CoV-2; COVID-19; genome sequencing; Spike glycoprotein; surveillance. 


\section{Introduction}

34 In December 2019, in China, an outbreak started due to a novel coronavirus strain that causes a severe illness, the coronavirus disease 2019 (COVID-19). The virus, SARS-CoV-2, has since spread globally [1]. Data reported to European Center for Disease Control show that clinical presentation of COVID-19 ranges from no symptoms to severe pneumonia, and that many cases have led to death [2]. According to available data, $32 \%$ of the diagnosed COVID-19 cases in the EU/EEA have been hospitalized, and 4\% thereof had severe form of illness [3]. Additionally, hospitalization rates have been significantly higher for adults 65 years and older [4]. The first

41 positive case of infection with SARS-CoV-2 in Romania was confirmed on $26^{\text {th }}$ of February

42 2020. With the ongoing spread of the virus, it is becoming critical to detect and classify the virus

43 in patient samples. As such, full genome characterization of this virus is instrumental for

44 updating diagnostics criteria and assessing viral evolution. To assess its genetic variation in a

45 South-Eastern European population, we herein generated genome sequences using metagenomic

46 sequencing, from 18 Romanian patients. We delineated the non-synonymous mutations and

47 constructed models of the protein structures from these sequences. We explicitly focused on the

48 spike (S) glycoprotein because it mediates infection of human cells, and is the main target of 49 most vaccine strategies and antibody-based therapeutics [5].

\section{Materials and Methods}

51 Collection and processing of samples

52 Nasopharyngeal and oropharyngeal swabs were collected from individuals from different

53 Romanian geographical areas. Total nucleic acid extraction was performed using the Maxwell®

54 RSC Viral Total Nucleic Acid Purification Kit (Promega, USA) as described by the 55 manufacturer. SARS-CoV-2 nucleic acid was detected using the E gene assay as the first-line 
screening tool, followed by confirmatory testing with the RdRp gene assay [6]. The RiboZero

57 Gold rRNA depletion protocol was used to remove human cytoplasmic and mitochondrial rRNA.

58 The total RNA quantity and integrity were measured with the Qubit RNA Assay Kit (Invitrogen,

59 Carlsbad, CA, USA). The TrueSeq Stranded Total RNA Library Prep Gold kit along with the

IDT for Illumina TruSeq RNA UD Indexes were used for sequencing-ready library preparation.

RNA fragmentation, first and second-strand cDNA synthesis, adenylation, adapter ligation and amplification were done according to the TruSeq Stranded Total RNA protocol. After amplification, the prepared libraries were quantified, pooled and loaded onto Illumina MiSeq DNA Sequencer. Sequence data from each sample was aligned to the SAR-CoV-2 reference genome (GenBank accession number: NC_045512.2) and variants called using SeqMan NGen (DNASTAR, Madison, WI, USA). Alignments were manually inspected to confirm variant calls and the viral sequence from each sample exported with SeqMan Pro (DNASTAR). Data mining for structures Atomic coordinates of the template, the novel SARS-CoV-2 spike protein in complex with Receptor Binding Domain (RBD) of the human Angiotensin-converting enzyme 2 (hACE2),

71 PDB: 6LZG, were retrieved from RCSB protein data bank. Spike protein sequences from our 72 genomes were modeled using I-Tasser [7] and the quality of models of Spike S proteins was 73 assessed with MolProbity [8]. Full length models were superimposed over template and root 74 mean square deviations (RMSD) of carbon alpha backbones were determined using PyMol 75 (PyMOL Molecular Graphics System, Version 2.0 Schrödinger, LLC).

76 Mutational analyses and molecular docking

77 Site Directed Mutator (http://marid.bioc.cam.ac.uk/sdm2/) was used to assess the impact of non78 synonymous single, double or triple mutations on the protein stability and to determine the $\Delta \Delta \mathrm{G}$ 
values for each protein. For mutations in the Spike protein binding site to RBD from ACE2, the difference in protein stability between Spike protein:RBD complex and Spike protein alone is indicated. For in silico docking, the HawkDock server (http://cadd.zju.edu.cn/hawkdock/) was used to conduct docking simulations between our modeled Spike S protein structures and ACE2. All molecular structures were visualized with PyMol.

\section{Results}

We performed real time RT-PCR tests on 32233 individuals, starting on 16.02.2020 until national testing relevant for this pandemic, valid at the time of writing.

Table 1. Aggregate of the testing data performed at Cantacuzino Military-Medical National ResearchDevelopment Institute (INCDMMC) and at national level [9]

\begin{tabular}{|c|c|c|}
\hline As of 22.06 .2020 & INCDMMC data & National data \\
\hline Total number of tests & 32233 & 626330 \\
\hline $\begin{array}{c}\text { Confirmed SARS-CoV-2 } \\
(\% \text { from total tested })\end{array}$ & $1630(5.05 \%)$ & $24045(3.83 \%)$ \\
\hline $\begin{array}{c}\text { Negative SARS-CoV-2 } \\
(\% \text { from total tested })\end{array}$ & $30603(94.95 \%)$ & $602285(96.17 \%)$ \\
\hline
\end{tabular}

92 A recent analysis of risk factors in Romanian patients was performed for a subset of 842 $\left.23^{\text {rd }}, 2020\right)$ [10]. For both sexes, patients with pre-existing cardiovascular conditions were 2.89 times more likely to succumb than patients who were not affected by this type of illness. Importantly, the next categories of patients who died were suffering from chronic renal diseases

97 (death more probable by 2.69) and diabetes (by 2.3 times). Given the high expression and circulating levels of hACE2 in the heart and kidneys, these findings support a mechanism

99 whereby Spike glycoprotein may attach to hACE2 in a dose-response manner. We also note that 
101 are not equally distributed between sexes, with men having the highest risks when suffering from

102 cancer or other immune deficiencies (Odds Ratio of 3.25), while women succumbing mostly

103 when suffering with chronic renal diseases (Odds Ratio of 4.3). This finding may reflect possible

104 gender differences in the levels and activity of ACE2, as has been observed in murine models $105[11]$

106

107 Genetic phylogeny

108 The sequences of Romanian SARS-CoV-2 sequences showed high ( 99.95 identity with Wuhan 109 seafood market pneumonia virus (Genbank accession number: NC_045512.2) and >99.6\% with 110 sequences from France, Germany, Greece, Italy, Poland, India, USA. Phylogenetic analysis

111 showed that the Romanian sequences belonged to different clusters (Figure 1). The following 112 sequences were introduced in GISAID (Global Initiative on Sharing All Influenza Data) under 113 accession numbers: EPI_ISL_445220, EPI_ISL_445243, EPI_ISL_447054, EPI_ISL_455468, 114 EPI_ISL_455469, EPI_ISL_455470, EPI_ISL_455471, EPI_ISL_455472, EPI_ISL_455473, 115 EPI_ISL_455474, EPI_ISL_455475, EPI_ISL_455476, EPI_ISL_455477, EPI_ISL_455479, 116 EPI_ISL_467779, EPI_ISL_467780, EPI_ISL_467781, EPI_ISL_467778. 
bioRxiv preprint doi: https://doi.org/10.1101/2020.06.28.175802; this version posted July 7, 2020. The copyright holder for this preprint (which was not certified by peer review) is the author/funder, who has granted bioRxiv a license to display the preprint in perpetuity. It is made available under aCC-BY-NC-ND 4.0 International license.

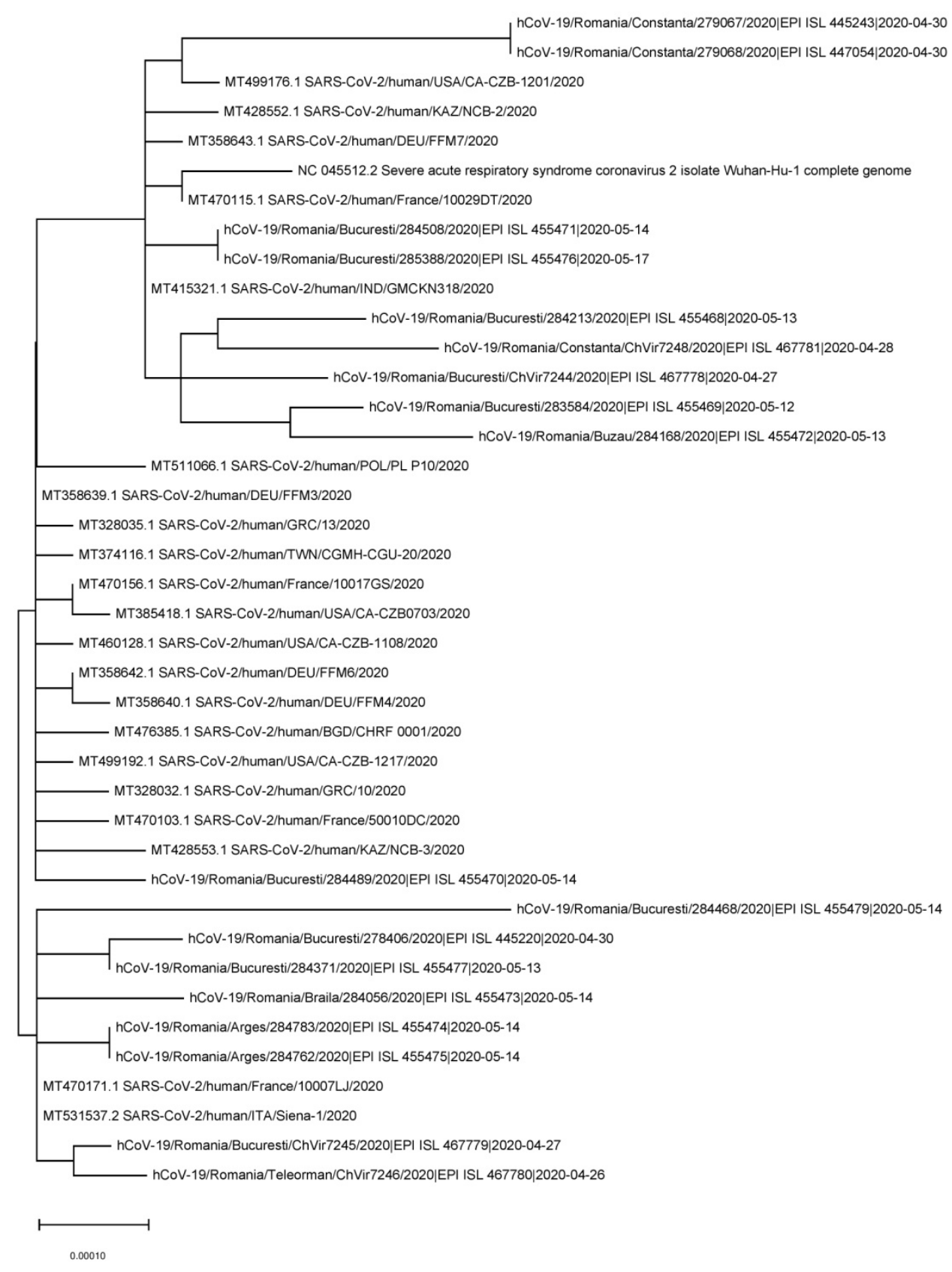

118 Fig. 1 Phylogenetic analysis of 18 SARS-CoV-2 complete genome sequences. The Wuhan reference genome from 119 GenBank accession number: NC_045512.2 and other European/American complete sequences are also shown from 120 different countries $(n=21)$. The tree was built by using the best fitting substitution model (HKY) through MEGA X 121 software.

122

\section{Molecular modeling}


129

130

131

132

133

134

135

136

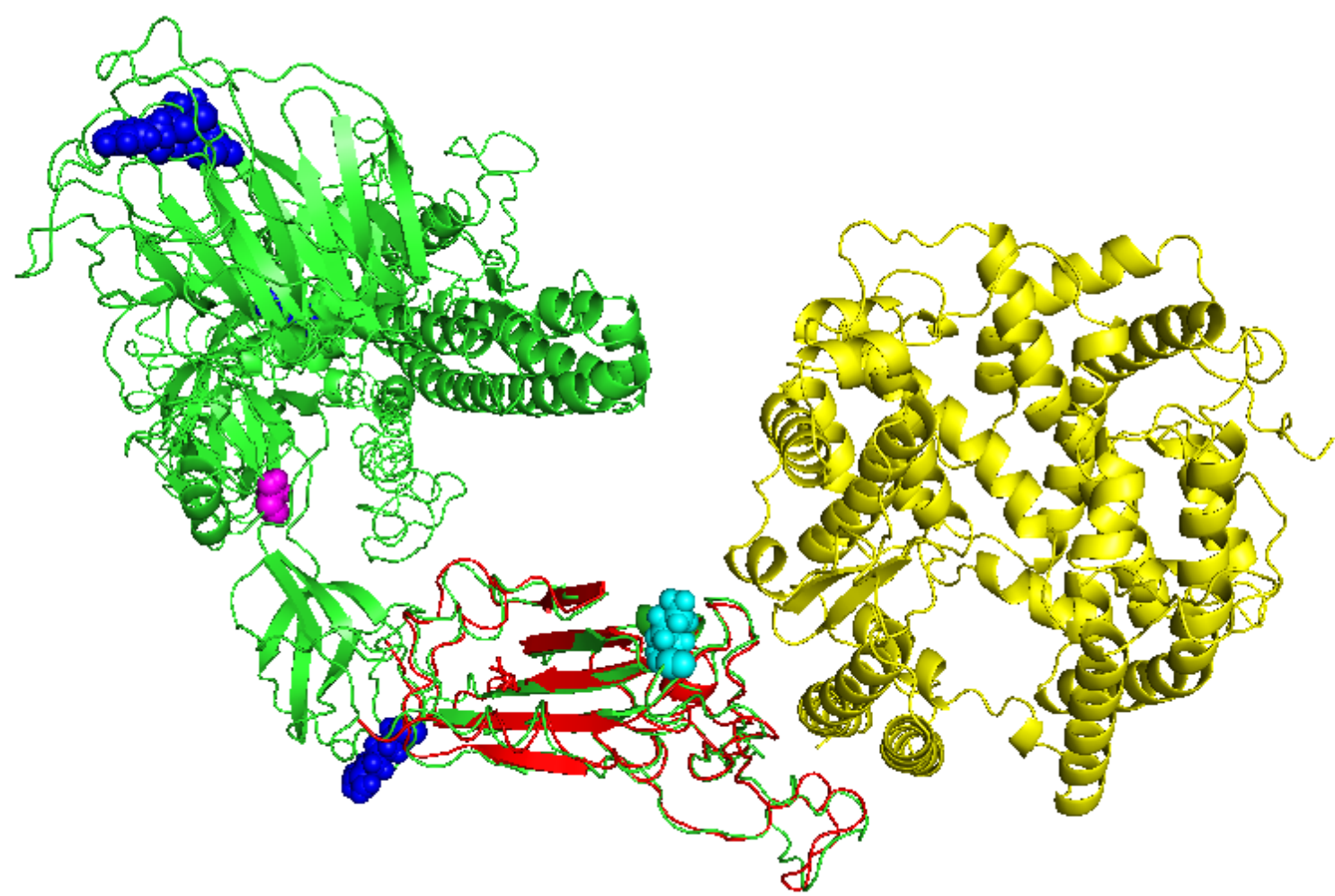

Figure 2: Docking of hACE2 (from PDB 6LZG, in yellow) with models of Spike glycoprotein variants (green) that contains the location of mutations described in this study (blue) with respect to binding partner hACE2. D614G mutation is shown in magenta; N439K mutation, that sits at the binding interface with hACE2 is shown in cyan. The structural overlap between RBD from Spike protein in PDB 6LZG and a constructed model of the mutant Spike protein is shown in red.

We used PDBsum to identify the critical amino acids at the binding interface, and further compiled with PyMol their identity (Table 2), where residues interfacing with multiple H-bonds are shown in bold. TM-scores [0-1] indicating the structural similarity to the overlapped model have been derived with I-TASSER (TM-Scores of 0.5 and above represent a high probability to match similar folds). The impact of the mutations on the stability of the complexes made with 
hACE2 is also indicated in Table 2. Although all mutants have negative (favorable) binding energy, compared to the native complex, only the triple mutant has a significantly higher value (60\% increase). We note that the double mutant $N 439 K, D 614 G$ that directly affects the binding interface, has only negligible positive effect on binding, but ranks above the rest of the variants.

Table 2. Key residues involved in the formation of interfaces between ACE2: Spike $S$ variants

\begin{tabular}{|c|c|c|c|c|}
\hline Spike Protein & Viral Interface Residues & $\begin{array}{c}\text { RMSD } \\
(\AA)\end{array}$ & $\begin{array}{l}\text { TM } \\
\text { score }\end{array}$ & $\begin{array}{c}\text { Binding free } \\
\text { energy - } \\
\text { complex } \\
\text { hACE2:variant } \\
\text { Spike proteins } \\
\text { (kcal/mol) }\end{array}$ \\
\hline PDB 6LZG & & - & - & -42.96 \\
\hline $\begin{array}{c}\text { Model SARS-CoV-2 RBD } \\
\text { [PDB: 6LZG] }\end{array}$ & $\begin{array}{l}\text { K417, G446, Y449, Y453, L455, F456, } \\
\text { A475, F486, N487, Y489, F490, Q493, } \\
\text { G496, Q498, T500, A501, G502, Y505 }\end{array}$ & - & - & - \\
\hline $\begin{array}{c}D 614 G \text { variant Spike S } \\
\text { overlapped to SARS-CoV-2 } \\
\text { RBD }\end{array}$ & Similar to SARS-CoV-2 RBD & 0.97 & 0.63 & -9.94 \\
\hline $\begin{array}{l}P 521 R, D 614 G \text { variant Spike S } \\
\text { overlapped to SARS-CoV-2 } \\
\text { RBD }\end{array}$ & Similar to SARS-CoV-2 RBD & 0.97 & 0.6 & -27.25 \\
\hline $\begin{array}{c}S 98 F, D 614 G \text { variant Spike S } \\
\text { overlapped to SARS-CoV-2 } \\
\text { RBD }\end{array}$ & Similar to SARS-CoV-2 RBD & 0.99 & 0.58 & -26.24 \\
\hline $\begin{array}{c}N 439 K, D 614 G \text { variant Spike S } \\
\text { overlapped to SARS-CoV-2 } \\
\text { RBD }\end{array}$ & $\begin{array}{c}\text { K417, K439, G446, Y449, Y453, L455, } \\
\text { F456, A475, F486, N487, Y489, F490, } \\
\text { Q493, G496, Q498, T500, A501, G502, } \\
\text { Y505 }\end{array}$ & 0.96 & 0.61 & -39.22 \\
\hline $\begin{array}{c}\text { V308L, D614G variant Spike S } \\
\text { overlapped to SARS-CoV-2 } \\
\text { RBD }\end{array}$ & Similar to SARS-CoV-2 RBD & 0.97 & 0.51 & -28.74 \\
\hline $\begin{array}{c}E 96 D, H 245 Y, D 614 G \text { variant } \\
\text { Spike S overlapped to SARS- } \\
\text { CoV-2 RBD }\end{array}$ & Similar to SARS-CoV-2 RBD & 0.98 & 0.6 & -67.21 \\
\hline
\end{tabular}


Mutations in the spike surface glycoprotein may be conducive to conformational changes, which can translate into changing antigenicity. As such, identifying the amino acids involved in conformational changes of the SARS-CoV-2 spike surface glycoprotein structure is essential to inform on patterns of mutations subject to positive selection. The role of detected mutations on overall thermodynamic stability of the protein variants is thus imperative, as shown in Table 3.

Table 3. Role of point mutations observed in the genomes on overall protein stability. $\Delta \Delta G$ parameter is a measure of the change in energy between the folded and unfolded states caused by point mutations [12].

\begin{tabular}{|c|c|c|c|}
\hline Variant Spike proteins & $\boldsymbol{\Delta} \boldsymbol{\Delta G}(\mathrm{kcal} / \mathrm{mol})$ & Effect on stability & $\begin{array}{c}\text { Solvent accessibility } \\
\text { compared to wild type }\end{array}$ \\
\hline S98F & -0.77 & destabilizing & $0.6 \%$ increase \\
\hline E96D & -1.34 & destabilizing & $9.2 \%$ increase \\
\hline H245Y & 0.78 & stabilizing & $25.8 \%$ increase \\
\hline V308L & -1.27 & destabilizing & $3.2 \%$ increase \\
\hline N439K & -0.2 & destabilizing & $20.7 \%$ increase \\
\hline P521R & 0.67 & stabilizing & $40.8 \%$ increase \\
\hline D614G & -0.58 & destabilizing & $23 \%$ increase \\
\hline
\end{tabular}

Spike $D 614 G$ variant has been the most widespread mutant encountered thus far in the 2020 genome datasets outside East Europe (3577 counts in GISAID database) [13]. D614G mutation is embedded into an immunodominant antibody epitope and is recognized by monoclonal antibodies isolated from recovered individuals, who had been infected with the original SARSindicates that the former is less stable thermodynamically, which translates into markedly

164 increased infectivity of ACE-2 expressing cells, consistent with epidemiological data. ${ }^{15}$

165 Furthermore, pseudoviruses containing both of these variants were neutralized with comparable efficiency by convalescent plasma [14]. However, another recent study demonstrated that Spike

167 variant $D 614 G$ significantly infects ACE-2 expressing cells, when compared to the native Spike 

evolve.

pseudovirus [16]. Given these factors, this mutant could mediate immune avoidance, although there is as-yet no definite clinical data to correlate the impact of this or any other mutation on infectivity. We note that none of the other mutations we describe have been found thus far in other cohorts, and that other mutations are themselves rare [13]. Tracking mutations in viral Spike glycoprotein continues to be paramount for vaccine and antibody therapy strategies that are currently being developed, and on evaluating new SARS-CoV-2 strains as they emerge and

\section{Acknowledgements:}

We are grateful to Luiza Ustea, Nicoleta Paraschiv and Adrian Cre $\square$ u for technical support. We thank the staff working from the Viral Respiratory Infections Laboratory (National Influenza Centre), colleagues from Cantacuzino Military-Medical Research and Development National Institute, the public health staff from the National Centre for Communicable Diseases Surveillance and Control, officials from the Ministry of Health and the Ministry of Defense.

Funding support was provided by Rompetrol Group NV (Fundatia pentru SMURD) and the Romanian Association for Shoulder and Elbow Surgery. The funding sources had no role in the study design, analysis or writing of report.

\section{REFERENCES}

[1] Zhou P, Yang XL, Wang XG, et al. A pneumonia outbreak associated with a new coronavirus of probable bat origin. Nature. 2020; 579(7798):270 273.

[2] Zheng F, Tang W, Li H, Huang YX, Xie YL, Zhou ZG. Clinical characteristics of 161 cases of corona virus disease 2019 (COVID-19) in Changsha. Eur Rev Med Pharmacol Sci. 2020; 24(6):3404ロ $\square 410$. 
[3] European Center for Disease Control: Rapid Risk Assessment: Coronavirus disease 2019 (COVID-19) in the EU/EEA and the UK- ninth update. Retrieved on June $8^{\text {th }}, 2020$.

[4] Garg S, Kim L, Whitaker M, et al. Hospitalization Rates and Characteristics of Patients Hospitalized with Laboratory-Confirmed Coronavirus Disease 2019 - COVID-NET, 14 States, March 1-30, 2020. MMWR Morb Mortal Wkly Rep. 2020; 69:458-464.

[5] Robson B. COVID-19 Coronavirus spike protein analysis for synthetic vaccines, a peptidomimetic antagonist, and therapeutic drugs, and analysis of a proposed achilles' heel conserved region to minimize probability of escape mutations and drug resistance. Comput Biol Med. 2020; 121:103749.

[6] Corman VM, Landt O, Kaiser M, et al. Detection of 2019 novel coronavirus (2019-nCoV) by real-time RT-PCR. Eurosurveillance. 2020; 25(3):2000045.

[7] Yang J, Zhang Y. I-TASSER server: new development for protein structure and function predictions. Nucleic Acids Research. 2015; 43: W174-W181.

[8] Williams CJ, et al. MolProbity: More and better reference data for improved all-atom structure validation. Protein Science. 2018; 27: 293-315.

[9] Informare COVID-19, Grupul de Comunicare Strategică, 13 Iunie 2020 (In Romanian). https://www.mai.gov.ro/informare-covid-19-grupul-de-comunicare-strategica-13-iunie-2020-ora13-00/. Retrieved 14.06.2020.

[10] Popovici O. Risk factors for death in confirmed cases of patients with COVID-19 (In Romanian). https://www.cnscbt.ro/index.php/analiza-cazuri-confirmate-covid19/1791-analizaepidemiologica-factori-de-risc-deces-cu-covid-19. Retrieved 09.06.2020.

[11] White MC, Fleeman R, Arnold AC. Sex differences in the metabolic effects of the reninangiotensin system. Biol Sex Differ. 2019; 10, 31.

[12] Kellogg EH, Leaver-Fay A, Baker D. Role of conformational sampling in computing mutation-induced changes in protein structure and stability. Proteins. 2011;79(3):830 $\square 838$.

[13] Korber B, et al. Spike mutation pipeline reveals the emergence of a more transmissible form of SARS-CoV-2. BioRxiv. 2020.04.29.069054; doi: https://doi.org/10.1101/2020.04.29.069054.

[14] Wang Q, et al. Immunodominant SARS Coronavirus Epitopes in Humans Elicited both Enhancing and Neutralizing Effects on Infection in Non-human Primates. ACS Infectious Diseases. 2016; 2, 361-376.

[15] Zhang L, Jackson CB, Mou H, Ojha1A, Rangarajan ES, Izard T, Farzan M, Choe H. The D614G mutation in the SARS-CoV-2 spike protein reduces S1 shedding and increases infectivity. BioRxiv. 2020.06.12.148726; doi: https://doi.org/10.1101/2020.06.12.148726.

[16] Hu J, He CL, Gao Q, Zhang GJ, Cao XX, Long QX, Deng HJ, Huang LY, Chen J, Wang K, Tang N, Huang AL. The D614G mutation of SARS-CoV-2 spike protein enhances viral infectivity and decreases neutralization sensitivity to individual convalescent sera. BioRxiv 2020.06.20.161323; doi: https://doi.org/10.1101/2020.06.20.161323. 